\title{
The value of CHA2DS2-VASc-HS score for prediction of adverse in- hospital outcomes in patients with non-ST segment elevation myocardial infarction
}

M.K.Mahmoud, H.M.Kabil, H.H.Ebed and A.M.Sabry

Cardiology, Dept., Faculty of Medicine, Benha Univ., Benha, Egypt

E-Mail:Majid.khalid21@fmed.bu.edu.eg

\begin{abstract}
For a treatment choice the early identification of non-ST myocardial infarction segment patients (NSTEMI) with poor in-hospital results is essential. The CHA2DS2-VASc-HS and CHA2DS2-VASc score was recently reported as a predictor of severity and poor outcomes in individuals with stable coronary artery disease (CAD) and acute coronary syndrome. Our research aimed at evaluating the precision of the CHA2DS2-VASc-HS score, which predicts poor outcomes in hospitals in NSTEMI patients. 120 NSTEMI patients have been enrolled. The score for CHA2DS2-VASc-HS has been computed. The participants of the research were split into two groups. Group I and Group II score $<4$ were placed in Patients with CHA2DS2-VASc-HS score>4. Patients with CHA2DS2-VASc-HS >4 were found to have higher unfavourable outcomes in the hospital than the CHA2DS2-VASc-HS score $<4$.
\end{abstract}

Keywords: Ischemic heart disease, CHA2DS2-VASc-HS score, adverse in-hospital outcomes, non-ST segment elevation myocardial infarction.

\section{Introduction}

The forecast in NSTEMI patients relies on several variables, including the degree of coronary blockage, left ventricular dysfunction, short-term danger associated with the blame and the presentation of the clinical condition [1].

NSTEMI patients usually have more comorbidities than STEMI patients. In NSTEACS patients, in-hospital mortality is $3-5 \%$. It is lower than STEMI, which accounts for $7 \%$. But death rates are fairly comparable at 6 months under both settings, which include about 12-13 percent. Long-term follow-up indicated that mortality rates were greater among NSTE-ACS patients than STEMI patients, with a double 4-year difference [2].

Risk assessment is essential for the treatment of NSTE-ACS patients. In NSTEACS patients the risk of morbidity and death varies according to risk factors, clinical characteristics and treatment approach. The current risk stratification recommendations of NSTE-ACS patients are recommended for use of thrombolysis in the risk score of Myocardial Infarction (TIMI) or in the GRACE score [3].

To assess risk in patients and suggest preventive measures, clinicians need easy, trustworthy, and quantitative methods. The TIMI and GRACE scoring methods used to stratify NSTE-ACS patients are based mainly on multivariate models that incorporate medical history components, admission ECG and cardiac biomarker data. [4]

The CHA2DS2-VASc score is a clinical predictor used to assess the risk of cardiac thromboembolism and advise anti-thrombotic treatment in non-valvular atrial fibrillation. The CHADS2 and CHA2DS2-VASc scores are extensively used in clinical practise and include comparable risk factors in coronary artery disease development (CAD). These ratings have shown a predictive significance in terms of the risk of mortality after a stroke, $\mathrm{CABG}$, and with stability of CAD and acute coronary syndrome [5].

CHA2DS2-VASc-HS score has recently been reported as a new $\mathrm{CAD}$ gravity predictor in stable CAD patients diagnosed by angiography. The CHA2DS2-VASc-HS nomenclature includes congestive cardiac insufficiency $(\mathrm{C})$, hypertension $(\mathrm{H})$, age $>75$ years (A2), diabetes mellitis (D) or stroke and TIA (S2), stroke (V), vascular disease (V) and male (sex), hyperlipidemia $(\mathrm{H})$ and smoking (S). This scoring method adds hyperlipidemia and smoking as additional significant risk factors for CAD compared to the CHA2DS2VASc scoring system, not just the use of men rather than females [6].

\section{Patients and methods}

This was a prospective cohort research conducted for one year at the Department of Cardiology, Benha University from May 2020 to May 2021.

All subjects received informed consent. A total of 120 NSTEMI patients have been chosen via purposeful sampling based on the specified criteria for registration.

NSTEMI has been characterised as having a usual chest pain over the last 48 hours and an ECG without a ST elevation that suggests myocardial ischemia and cardiac enzymes. [7] 
Cardiac troponin I was collected with a threshold of $1.0 \mathrm{ng} / \mathrm{ml}$ as a positive biomarker.

Patients with cardiac valves, congenital cardiomyopathy, myocarditis, pericarditis, severe co-morbidities and revascularization (PCI or $\mathrm{CABG}$ ) were eliminated during hospitalization by index.

Demographic features, including age, sex, diabetes mellitus, hypertension, hyperlipidemia, current smoking cigarettes, family history of premature $\mathrm{CAD}$, chronic cardiac disorder, preceding ischemic or transient stroke ischemic (TIA), peripheral artery disease (PAD), presenting symptoms and biochemical and Echocardiography data(assessment of LV global longitudinal strain (GLS) and ejection fraction by Simpson method) were obtained.

The score of CHA2DS2-VASc-HS was then computed and the research participants split into two groups. CHA2DS2-VASc-HS > 4 patients are classified in group I and $<4$ in group II.

DM was diagnosed as a fasting blood glucose $>126 \mathrm{mg} / \mathrm{dL}$ or the current use of antidiabetic medications. [8] HTN was diagnosed if repeated measurements of systolic and diastolic blood pressure were $>140 \mathrm{~mm} \mathrm{Hg}$ and $>90 \mathrm{~mm} \mathrm{Hg}$, respectively, or if the patient received chronic anti-hypertensive medication treatment.[9] A level of low-density lipoprotein cholesterol above $160 \mathrm{mg} / \mathrm{dL}$ according to the National Cholesterol Education Program Adult Treatment Panel III recommendations or the usage of lipid lowering medications was defined as HL.[10] Cigarette smoking was defined as smoking a minimum of 10 cigarettes per day for at least 1 year in patients who had never stopped smoking before the day of evaluation.[11] Family history was defined as the presence of ischemic heart disease or sudden cardiac death in a male first degree relative aged $<55$ years or in a female first- degree relative aged $<65$ years. Chronic heart failure was defined as Killip Classification. [12] Vascular disease was considered to be the presence of PAD where at least $50 \%$ stenosis diagnosed by Dupplexsonography of the non- coronary artery circulation. [13]

The results were then examined and documented in the hospital. Recent ischemia, heart failure, cardiovascular shock, important arrhythmias and mortality were the outcome factors. Overall unfavourable results were characterised as one of many outcomes that is categorised as an outcome. The composite outcome or overall unfavourable outcomes were evaluated in patients that had suffered any of the specific events indicated by elements in the results variables. [14]

\section{Results and discussion}

A total of 120 patients were studied, including $78(65 \%)$ male and $42(35 \%)$ female (Fig 1). Patients having CHA2DS2-VASc-HS score $>4$ were assigned as group I and patients having CHA2DS2-VASc-HS score $\square 4$ were assigned as group II. Baseline characteristics are mentioned in Table (1).

In-hospital adverse events are reported in Table II and III. Cardiogenic shock $(\mathrm{p}=0.04 \mathrm{~s})$, heart failure $(\mathrm{p}=0.03 \mathrm{~s})$ and recurrent ischemia $(\mathrm{p}=0.04 \mathrm{~s})$ were significantly higher with CHA2DS2-VASc-HS score $>4$. The significant arrhythmia and death occurred in group I and none in group II. $20 \%$ of 60 patients in group I experienced combined adverse in-hospital outcomes, on the contrary $3.3 \%$ of the patients in group II which was significantly higher $(p=0.01)$ with $R R=6$. The prediction by the area under the ROC curves for CHA2DS2VASc-HS score>4 was good with accuracy $89 \%$ (Fig. 2) with sensitivity $85.7 \%$ and specificity $54.7 \%$ Table (4).

Table (1) Baseline characteristics of study population $(\mathrm{N}=120)$.

\begin{tabular}{lccc}
\hline Variables & $\begin{array}{c}\text { Group I }(\mathbf{n}=\mathbf{6 0}) \\
\text { Mean } \pm \text { SD }\end{array}$ & $\begin{array}{c}\text { Group II (n= 60) } \\
\text { Mean } \pm \text { SD }\end{array}$ & p value \\
\hline S. creatinine (mg/dl) & $1.25 \pm 0.31$ & $1.13 \pm 0.30$ & $0.19 \mathrm{~ns}$ \\
RBS (mg/dl) & $8.4 \pm 4.8$ & $7.3 \pm 2.6$ & $0.14 \mathrm{~ns}$ \\
Cardiac troponin I (ng/dl) & $4.0 \pm 2.3$ & $3.1 \pm 2.2$ & $0.03 \mathrm{~S}$ \\
Total Cholesterol( mg/dl) & $203.5 \pm 35.6$ & $198.6 \pm 30.2$ & $0.26 \mathrm{~ns}$ \\
Triglyceride (mg/dl) & $200.8 \pm 40.9$ & $170.2 \pm 32.5$ & $0.27 \mathrm{~ns}$ \\
LDL cholesterol (mg/dl) & $112.1 \pm 30.2$ & $98.6 \pm 11.2$ & $<0.02 \mathrm{~s}$ \\
HDL cholesterol (mg/dl) & $39.4 \pm 6.4$ & $42 . \pm 4.6$ & $0.22 \mathrm{~ns}$ \\
ST segment deviation in ECG & 28 & 22 & $0.22 \mathrm{~ns}$ \\
Ejection fraction (percent) & $53.2 \pm 6.3$ & $56.9 \pm 7.1$ & $0.03 \mathrm{~s}$ \\
\hline
\end{tabular}


Table (2) Adverse in-hospital outcomes variables of the study population $(\mathrm{N}=120)$.

\begin{tabular}{lccccccc}
\hline Outcomes variables & \multicolumn{2}{c}{ Group I $(\mathbf{n}=\mathbf{6 0})$} & \multicolumn{2}{c}{ Group II $(\mathbf{n}=\mathbf{6 0})$} & \multicolumn{2}{c}{ Total $(\mathbf{N}=120)$} & p value \\
& Number & \% & Number & \% & Number & \% & \\
\hline Cardiogenic shock & 6 & 10.0 & 1 & 1.7 & 7 & 5.8 & $0.04 \mathrm{~s}$ \\
Heart failure & 10 & 16.7 & 2 & 3.3 & 12 & 10 & $0.03 \mathrm{~s}$ \\
Recurrent ischemia & 7 & 11.7 & 2 & 3.3 & 9 & 7.5 & $0.04 \mathrm{~s}$ \\
Significant arrhythmia & 1 & 1.7 & 0 & 0.0 & 1 & 0.8 & $0.97 \mathrm{~ns}$ \\
Death & 2 & 3.3 & 0 & 0.0 & 2 & 1.7 & $0.47 \mathrm{~ns}$ \\
\hline
\end{tabular}

Table (3) Combined adverse in-hospital outcome among the study population $(\mathrm{N}=120)$.

\begin{tabular}{lccccc}
\hline Group & $\begin{array}{c}\text { Adverse in-hospital } \\
\text { outcome }\end{array}$ & \multicolumn{2}{c}{$\begin{array}{c}\text { Adverse in-hospital } \\
\text { outcome }\end{array}$} & p value \\
& Present & \% & Absent & \% & RR \\
\hline Group I (n= 60) & 12 & 20.0 & 48 & 80.0 & $0.01 \mathrm{~s}$ \\
Group II (n= 60) & 2 & 3.3 & 58 & 96.7 & \\
Total $(\mathbf{n}=\mathbf{1 2 0})$ & 14 & 11.7 & 106 & 88.3 & \\
\hline
\end{tabular}

Table (4) Performance test of CHA2DS2-VASc-HS score in the setting of NSTEMI (N=120).

\begin{tabular}{|c|c|c|c|c|c|}
\hline \multirow[t]{2}{*}{$\mathrm{CHA}_{2} \mathrm{DS}_{2}$-VASc -HS score } & \multicolumn{2}{|c|}{ Adverse in-hospital outcomes } & \multirow[t]{2}{*}{ Sensitivity } & \multirow[t]{2}{*}{ Specificity } & \multirow[t]{2}{*}{ Accuracy } \\
\hline & Occurred & Not occurred & & & \\
\hline High: score>4 & 12 & 48 & $85.7 \%$ & $54.7 \%$ & $89 \%$ \\
\hline Low: scored $<4$ & 2 & 58 & & & \\
\hline Total & 14 & 106 & & & \\
\hline
\end{tabular}

Table (5) Area Under the Curve: Test Result Variable(s): Predicted probability.

\begin{tabular}{ccccc}
\hline Area & Std. Errora & $\begin{array}{c}\text { Asymptotic } \\
\text { Sig.b }\end{array}$ & \multicolumn{2}{c}{$\begin{array}{c}\text { Asymptotic 95\% } \\
\text { Confidence Interval }\end{array}$} \\
\hline & & & Lower Bound & Upper Bound \\
.899 & .032 & .000 & .836 & .961 \\
\hline
\end{tabular}

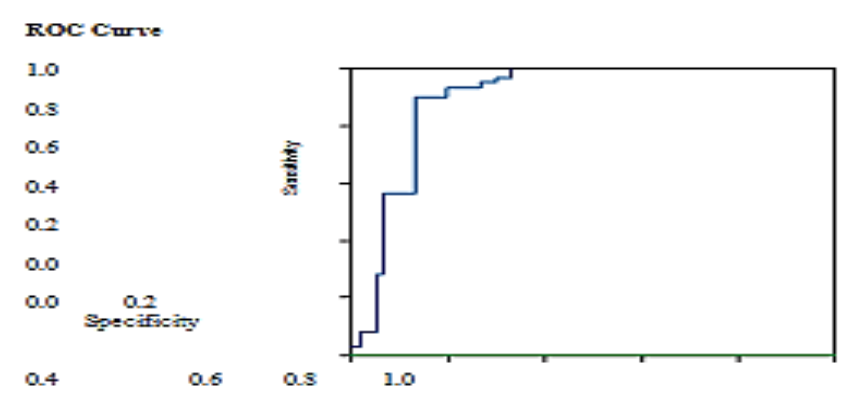

Fig. (1) Receiver operating characteristic (ROC) curves for $\mathrm{CHA}_{2} \mathrm{DS}_{2}-\mathrm{VASc}-\mathrm{HS}$ score $>4$ in the prediction of occurring adverse in-hospital outcomes.

The primary aim of the research was to identify a combination of CHA2DS2-VAScHS with unfavourable outcomes in hospitals in patients with non-ST myocardial infarction elevation (NSTEMI) segment. The inclusion and exclusion criteria comprised a total of 120 individuals with NSTEMI, both of gender and all ages.

Male patients comprised mostly 65 percent in the study population. Women's patients were $35 \%$. Similar male predominance was observed in virtually all research linked to coronary artery disease (CAD). As women are paid less attention and the access to health services for them is restricted, especially in the poor socio-economic population, as this male prevalence may contribute in our nation.

There was no statistically significant difference in clinical parameters across the trial groups. We discovered that 46.6 percent of ST patients in group I was higher than in group II (41.6 percent). A cross-sectional 
research was performed at NICVD in Bangladesh in 2011. The degree of STsegment depression is clearly related to the severity of coronary artery disease. [15]

The study population average left ventricular ejection fraction (LVEF) was 54.6 \pm 7.1. In Group I (53.2 \pm 6.3$)$ LVEF dropped from Group II $(56.9 \pm 7.1)$ with statistically significant difference. The recent NICVD research in NSTEMI patients showed a mean LVEF of $54.8 \pm 2.1$. [16] Lower LVEF may be responsible for more risk factors and severe illness in Group II.

The mean LDL $(112.1 \pm 30.2)$ cholesterol in the Lipid profile was found to be substantially higher in Group I. High LDL has been recognized as a significant cause of cardiovascular atherosclerotic disease (ASCVD). Taoolar et al. observed LDL cholesterol mean to be $112.1 \pm 43$. The total level of cholesterol and triglyceride was greater in Group II than in Group I, although not significant $(p>0.05)$. [17] HDL cholesterol in group I was lower than in group II with no significant difference.

Of biochemical markers, only the amount of cardiac troponin was observed in group I greater than in group II and was significant. Cohen also discovered that elevated troponin I is a separate indicator for adverse events. However, there is no significant difference between two groups in serum creatinine and RBS.

Among NSTEMI patients with CHA2DS2-vASc-HS >4 (Group I), 16.7\% had heart failure, $11.7 \%$ had recurring ischemia, and $10 \%$ had cardiogenic shock. Only $1.7 \%$ got major arythmias and $3.3 \%$ died in Group II. 3.3 percent, respectively 3.3 percent and 1.7 percent experienced heart failure, recurrent ischemia and severe arhythmias for CHA2DS2-VASc-HS patients, respectively 4 (Group II). Heart failure, cardiogenic shock and recurring ischemia have been more significant in Group I than Group II $(\mathrm{p}<0.04)$. Islam carried performed a NICVD research on NSTEMI patients that corresponds to the unfavourable results of this study. [16] The same research revealed a total of $6.4 \%$ recurring ischemistry, 5\% cardiogenic shock and $1.4 \%$ of patients died, compared with $7.5 \%, 5.8 \%$ and $1.7 \%$ recurring ischemia, cardiogenic shock and mortality. Another research revealed that recurrent ischemia in hospitals with NSTEMI was 10 percent, 18 percent heart failure and 6 percent mortality. [18]

With regard to all patients who had bad outcomes in the hospital, $20 \%$ of patients in Group I had adverse outcomes in the hospital, whereas in Group II $3.3 \%$ had poor outcomes in hospitals and the difference was statistically significant. Some people have had more than one bad result in the hospital. In a comparable Taoolar research, et al. discovered 23.2 percent in patients with cardial events $>4$ compared to 3.8 percent in patients with CHA2DS2-VASc$\mathrm{HS}>4.17$. The results of this research corresponded to the prior study. Risk was evaluated according to relative risk (RR). A relative risk in hospital outcome development (RR) of greater than 1, thus CHA2DS2-VASc$\mathrm{HS}>4$ is a risk factor. The recipient operating feature curve (ROC) analysis found the optimum cutoff for CHA2DS2 - VASc-HS >4 to predict cardiac outcomes, with 85.7 percent sensitivity and 54.7 percent speciality. The forecast was thus very excellent at 89 percent accuracy.

CHA2DS2-VASc-HS has recently been transformed into a novel developing CAD severity predictor in steady CAD patients and CHA2DS2-VASc in ACS patients. No research of this CHA2DS2-VASc-HS score was carried out in our nation to predict hospital outcomes in NSTEMI patients. In this research, CHA2DS2-VASc-HS was intended to be a risk factor that would predict poor hospital outcomes following NSTEMI.

Simple risk ratings are favoured in clinical practise. The ideal score should be calculated easily and simple for quick screening of highrisk individuals to avoid adverse events. The results of high-risk patients can be predicted with comorbidity findings using the CHA2DS2-VASc-HS scoring system without the need for information on vital admission signals, which are user-friendly and timesaving, requires software to calculate the total risk assessment and does not involve any additional costs. As a prognostic predictor for poor outcomes in hospitalised NSTEMI patients, CHA2DS2-VASc-HS scoring may thus play an essential role.

\section{Conclusion}

CHA2DS2-VASc-HS scores may be used to predict the probability of unfavourable clinical events in NSTEMI patients. We are able to pay particular attention to individuals at greater risk with CHA2DS2-VASc-HS $>4$, and develop an optimal treatment plan to minimise risks of future adverse events. In conclusion, our research has shown that greater unfavourable hospital outcomes have been seen in individuals with high CHA2DS2VASc-HS.

\section{References}

[1] J.A. Lemos, R. O'Rourke, R.A. Harrington. Unstable Angina and Non-ST-Segment Elevation 
Myocardial Infarction. In: V Fuster, RA Walsh, RA Harrington, eds. Hurst's The Heart, vol. 13 New York: Mc Graw Hill, pp. 1328-1332, 2011.

[2] C.W. Hamm, J.P. Bassand, S. Agewall, J. Bax, E. Boersma, H. Bueno, et al. ESC Guidelines for the management of acute coronary syndromes in patients presenting without persistent ST-segment elevation: The Task Force for the management of acute coronary syndromes (ACS) in patients presenting without persistent STsegment elevation of the European Society of Cardiology (ESC). Eur Heart J vol. 32(23), pp. 2999-3054, 2011.

[3] K.A. Eagle, M.J. Lim, O.H. Dabbous, K.S. Pieper, R.J. Goldberg, F. Van de Werf, et al. A validated prediction model for all forms of acute coronary syndrome: estimating the risk of 6month post discharge death in an international registry. JAMA; vol. 291(22), pp. 2727-2733, 2004.

[4] E.M. Antman, M. Cohen, P. Bernink, C.H. McCabe, T. Horacek, G. Papuchis, et al. The TIMI Risk Score for Unstable Angina/Non-ST Elevation MIA Method for Prognostication and Therapeutic Decision Making. JAMA vol. 284(7), pp. 835-842, 2000.

[5] D. Poci, M. Hartford, T. Karlsson, J. Herlitz, N. Edvardsson, K. Caidahl. Role of the CHADS2 score in acute coronary syndromes: risk of subsequent death or stroke in patients with and without atrial fibrillation. Chest vol. 141(6), pp. 1431-1440, 2012.

[6] M. Çetin , M. Çakýcý , M. Zencir , H. Tapolar, E. Baysal, M. Ballý, et al. Prediction of coronary artery disease severity using CHADS2 and CHA2DS2-VASc scores and a newly defined CHA2DS2-VASc-HS score. Am J Cardiol; vol. 113(6): pp. 950956, 2014.

[7] K. Hygesen, J.S. Alpert, A.S. Jaffe, M.L. Simoons, B.R. Chaitman, H.D. White, et al. Definition of myocardial infarction. Eur Heart J. vol. 33, pp. 2551-2567, 2012.

[8] S. Genuth, K.G. Alberti, P. Bennett, J. Buse, R. Defronzo, R. Kahn, et al. Expert Committee on the Diagnosis and Classification of Diabetes Mellitus. Follow-up report on the diagnosis of diabetes mellitus.
Diabetes Care; vol. 26, pp. 31603167, 2003.

[9] G. Mancia, R. Fagard, K. Narkiewicz, J. Redon, A. Zanchetti, M. Bohm, et al. $2013 \mathrm{ESH} / \mathrm{ESC}$ guidelines for the management of arterial hypertension: the Task Force for the Management of Arterial Hypertension of the European Society of Hypertension (ESH) and of the European Society of Cardiology (ESC). Euro Heart J; vol. 34, pp. 2159-2219, 2013.

[10] S.M. Grundy, N.J. Stone, A.L. Bailey, et al. 2019 AHA/ACC guideline on the management of blood cholesterol: a report of the American College of Cardiology/American Heart Association Task Force on Clinical Practice Guidelines. Circulation; vol. 139, pp. 1082-1143, 2019.

[11]U. Mons, A. Müezzinler, C. Gellert, et al. Impact of smoking and smoking cessation on cardiovascular events and mortality among adults: metaanalysis of individual participant data from prospective cohort studies of the CHANCES consortium. BMJ; vol. 350, pp. 1551, 2015.

[12]T. Killip, J. Kimball. Treatment of myocardial infarction in coronary care unit: a two year experience with 250 patients. Am J Cardiol; vol.20, pp. 457-465, 1976.

[13] R. Collins, J. Burch, G. Cranny, R. Guiar-Ibanez, D. Craig, K. Wright et al. Duplex ultrasonography, magnetic resonance angiography, and computed tomography angiography for diagnosis and assessment of symptomatic, lower limb peripheral arterial disease: systematic review. BMJ.; vol. 334, pp. 1257, 2017.

[14]G. Cordoba, L. Schwartz, S. Woloshin, H. Bae, P.C. Gøtzsche. Definition, reporting, and interpretation of composite outcomes in clinical trials: systematic review. BMJ; vol. 341(c), pp. 3920, 2010.

[15] M.E. Miah, A.H. Chowdhury, K.Q. Islam, M. Jamaluddin, S. Ghafur, M. Khalequzzaman, et al. ST Segment Depression in 12 lead ECG and Severity of Coronary Artery Disease in Non-ST segment elevation Acute Coronary Syndrome. Cardiovasc J. vol. 9(2), pp. 116-121, 2017.

[16] Islam Akmr and A.A. Majumder. Coronary artery disease in Bangladesh: a review. Indian Heart J. vol. 65(4), pp. 424-435, 2013. 
[17]H. Tapolar, M. Çetin, M. Ballý, B. Adil, Y.B. Ömür, S.O. Türkmen et al. CHA2DS2-VASc-HS score in nonST elevation acute coronary syndrome patients: assessment of coronary artery disease severity and complexity and comparison to other scoring systems in the prediction of in-hospital major adverse cardiovascular events. Anatol J Cardiol; vol. 16(10), pp. 742-748, 2016.

[18] P.G. Steg, R.J. Goldberg, J.M. Gore, K.A. Fox, K.A. Eagle, M.D. Flather, et al. Baseline characteristics, management practices, and in-hospital outcomes of patients hospitalized with acute coronary syndromes in the Global Registry of Acute Coronary Events. Am J Cardiol vol. 90(4), pp. 358-363,2002. 